\title{
Biologia e parasitismo de Trichogramma atopovirilia Oatman \& Platner e Trichogramma pretiosum Riley (Hymenoptera, Trichogrammatidae) em ovos de Spodoptera frugiperda (J.E. Smith) (Lepidoptera, Noctuidae)
}

\author{
Eduardo B. Beserra ${ }^{1} \&$ José Roberto P. Parra ${ }^{2}$
}

\begin{abstract}
'Departamento de Farmácia e Biologia, Universidade Estadual da Paraíba. Caixa Postal 781/791, 58100-000 Campina Grande-PB, Brasil.

2Departamento de Entomologia, Fitopatologia e Zoologia Agrícola, Escola Superior de Agricultura "Luiz de Queiroz", Universidade de São Paulo. Caixa Postal 09, 13418-900 Piracicaba-SP, Brasil. Endereço eletrônico: jrpparra@esalq.usp.br
\end{abstract}

\begin{abstract}
Biology and parasitism of Trichogramma atopovirilia Oatman \& Platner and Trichogramma pretiosum Riley (Hymenoptera, Trichogrammatidae) on eggs of Spodoptera frugiperda (J.E. Smith) (Lepidoptera, Noctuidae). The parasitism capacity and development of Trichogramma atopovirilia Oatman \& Platner and Trichogramma pretiosum Riley were studied in order to select the most suitable species to control Spodoptera frugiperda (J. E. Smith). The experiments were conducted under laboratory conditions at $25 \pm 2^{\circ} \mathrm{C}, 70 \pm 10 \% \mathrm{RH}$ and $14 \mathrm{~L}: 10 \mathrm{D}$ photoperiod. Both $T$. atopovirilia and $T$. pretiosum showed good development and adaptation to $S$. frugiperda eggs even after being reared for several generations in eggs of Anagasta kuehniella Zeller. However, T. atopovirilia females were more aggressive and showed higher specificity to the pest, with a higher parasitism capacity in eggs laid with different physical barriers and were more accepted by the natural host in comparison with A. kuehniella. Because of the higher parasitism rate and specificity of $T$. atopovirilia to the pest's egg this species should be given preference to control S. frugiperda.
\end{abstract}

KeYwords. Biological control; fall armyworm; host suitability; parasitoids; trichogrammatid.

Resumo. Comparou-se a capacidade de paratisimo e o desenvolvimento de Trichogramma atopovirilia Oatman \& Platner e T. pretiosum Riley visando à seleção da espécie mais adequada para o controle de Spodoptera frugiperda (J. E. Smith). Os experimentos foram conduzidos em laboratório à temperatura de $25 \pm 2^{\circ} \mathrm{C}$, U.R. de $70 \pm 0 \%$ e fotoperíodo de $14 \mathrm{~L}: 10 \mathrm{E}$. Tanto T. atopovirilia como T. pretiosum desenvolveram-se bem e mostraram-se adaptados a ovos de S. frugiperda, mesmo após serem criados por várias gerações em ovos de Anagasta kuehniella Zeller. Entretanto, fêmeas de $T$. atopovirilia foram mais agressivas e de maior especificidade à praga, já que apresentaram maior capacidade de parasitismo em posturas com diferentes barreiras físicas e maior aceitação pelo hospedeiro natural, em relação à A. kuehniella. Por este maior parasitismo e especificidade de T. atopovirilia aos ovos da praga, deve-se dar preferência à utilização desta espécie para controle de $S$. frugiperda.

Palavras-Chave. Adequação hospedeira; controle biológico; lagarta-do-cartucho; parasitóides; tricogramatídeo.

A lagarta-do-cartucho, Spodoptera frugiperda (J.E. Smith, 1797), é uma praga de grande importância econômica nas regiões do Brasil onde se cultiva o milho (BERTELs 1970). Seu controle é feito com a aplicação de inseticidas que, em muitos casos, não solucionam o problema (CoRTEZ \& TRUILlLo 1994) e causam impactos negativos, como a contaminação ao meio ambiente, problemas de resistência da praga e desequilíbrio biológico. Nos últimos anos, tem-se pesquisado a possibilidade de controle de $S$. frugiperda através de liberações de Trichogramma atopovirilia Oatman \& Platner, 1983 e Trichogramma pretiosum Riley, 1879, parasitóides frequientes dessa praga (Resende \& Ciociola 1996). Porém, faltam estudos que mostrem a capacidade dessas espécies em parasitar e se desenvolver nos ovos da praga e que dêem uma indicação da eficiência dessas espécies como agentes de controle biológico de $S$. frugiperda.

Parasitóides de ovos do gênero Trichogramma constituem um importante grupo de inimigos naturais com potencial para o controle biológico, já que eliminam a praga antes que qualquer dano seja causado à cultura (BLEICHER \& PARRA 1989; BOTELHO et al. 1995). O grande número de espécies de Trichogramma apresenta diferenças que podem alterar sua eficiência no controle de uma determinada praga. Essas espécies variam, em sua preferência por hospedeiros (reconhecimento e aceitação), no comportamento de busca (localização do hábitat, localização hospedeira) e tolerância às condições ambientais (HASSAN \& Guo 1991; WüHRER \& HASSAN 1993). Dependem, ainda, da qualidade do hospedeiro como alimento para as suas larvas (SснміDт \& Sмiтн 1985), sendo que seu ciclo de vida pode ser alterado em função do tipo de hospedeiro.

A capacidade do parasitóide de atacar e se desenvolver no hospedeiro é uma etapa crítica em um programa de controle biológico, desde que o hospedeiro possua alguma característica que evite a oviposição ou iniba o desenvolvimento do parasitóide (NAVARRo \& MARCANo 1999) e com isto diminua a sua eficiência de controle. Para o sucesso de um programa de controle biológico é importante a escolha adequada de uma espécie de inimigo natural a ser utilizada. Para tanto, são necessárias avaliações que devem envolver, principalmente, a preferência e a adequação hospedeira, para que se selecione uma espécie que seja eficiente ao controle da praga (HASSAN 1997). Assim, a presente pesquisa tem como 
objetivo comparar o desenvolvimento e a capacidade de parasitismo de $T$. atopovirilia e $T$. pretiosum visando selecionar a espécie mais indicada para o controle biológico de S. frugiperda.

\section{MATERIALE MÉTODOS}

O experimento foi conduzido no Laboratório de Biologia de Insetos do Departamento de Entomologia, Fitopatologia e Zoologia Agrícola da Escola Superior de Agricultura "Luiz de Queiroz", Universidade de São Paulo, à temperatura de $25 \pm 2^{\circ} \mathrm{C}$, U.R. de $70 \pm 10 \%$ e fotoperíodo de 14L:10E. Foram utilizadas 40 fêmeas recém-emergidas de $T$. atopovirilia e $T$. pretiosum (mantidas por várias gerações em laboratório) individualizadas em tubos de vidro (12,0 x 75,0 mm), oferecendo-se, para cada fêmea, 40 ovos de $S$. frugiperda com, no máximo, 12 horas de idade e, como fonte de alimento, uma gota de mel puro. Antes da instalação do experimento, as fêmeas de cada espécie foram mantidas, por uma geração, em ovos de S. frugiperda, procurando-se, dessa forma, eliminar o efeito do hospedeiro alternativo, pelo provável condicionamento pré-imaginal. Visando avaliar apenas a qualidade do hospedeiro para o desenvolvimento de $T$. atopovirilia e T. pretiosum, os ovos de $S$. frugiperda foram separados, eliminando-se, dessa forma, as barreiras físicas (camadas e escamas) de suas posturas. Inicialmente, as posturas foram imersas por 15 minutos em água destilada, para facilitar a separação dos ovos, e estes, em seguida, destacados com pincel e fixados à cartolina de coloração azul, por meio de água destilada. O parasitismo foi permitido por 48 horas, avaliando-se: duração do desenvolvimento (ovo-adulto), porcentagem de emergência, número de ovos parasitados, porcentagem de parasitismo, número de adultos emergidos por ovo, longevidade das fêmeas e razão sexual (rs = fêmea/fêmea + macho).

A capacidade de parasitismo de T. atopovirilia e $T$. pretiosum foi avaliada individualmente, utilizando-se posturas recém colocadas ( $0-12$ horas) de $S$. frugiperda, com diferentes características físicas, a saber: tratamento 1 , uma camada de ovos sem escamas; tratamento 2, duas camadas de ovos sem escamas; tratamento 3, uma camada de ovos com baixa densidade de escamas; tratamento 4, uma camada de ovos com alta densidade de escamas. Foram individualizadas, em tubos de vidro (12,0 x 7,0 mm), para cada tipo de postura, 15 fêmeas dessas espécies alimentadas com uma gota de mel puro. O parasitismo foi permitido por 48 horas, removendo-se, após este período, as fêmeas dos tubos de criação. O número de ovos de cada postura foi contado com base no número de lagartas eclodidas e de ovos parasitados. Quando da emergência dos adultos de Trichogramma, foram contados: número de ovos com orifício de saída e ovos escuros sem a emergência de adultos, avaliando-se, assim, o número de ovos parasitados e a porcentagem de parasitismo.

Os tamanhos das fêmeas de Trichogramma spp., com base no comprimento da tíbia posterior (BAI et al. 1992; OLSON \& ANDOw 1998), e do ovipositor, foram comparados utilizandose 20 fêmeas de T. atopovirilia e T. pretiosum, provenientes de ovos de S. frugiperda e de Anagasta kuehniella (Zeller). As fêmeas foram mortas e clarificadas em ácido acético glacial por 48 horas e, a seguir, montadas em lâminas com "Hoyers" (KAZMER \& LucK 1995) e secas em estufa a $60^{\circ} \mathrm{C}$. O comprimento da tíbia posterior foi considerado a partir da articulação com o fêmur até a junção com o tarso (Olson \& AnDow 1998), e do ovipositor, a partir da base até o seu ápice (Fig. 1). Para se correlacionar o tamanho da tíbia com o comprimento do ovipositor, as medições dessas estruturas foram feitas em uma mesma fêmea através de uma ocular micrométrica acoplada ao estéreomicroscópio com capacidade de aumento de até 100 vezes.

A preferência hospedeira foi avaliada adotando-se a metodologia de Hassan (1989). Ovos de A. kuehniella e $S$. frugiperda foram distribuídos em diagonal, em pequenas áreas $\left(0,4 \mathrm{~mm}^{2}\right)$ de uma arena de cartolina azul $\left(20 \mathrm{~mm}^{2}\right)$ colocada no interior de um tubo de vidro $(8,5 \times 2,5 \mathrm{~cm})$, no centro da qual foi oferecida uma gota de mel puro. Fêmeas $(\mathrm{n}=20)$ de $T$. atopovirilia e $T$. pretiosum recém emergidas de ovos de $A$. kuehniella, foram individualizadas no interior dos tubos e monitoradas a cada 5 min, durante um período de 6 horas, registrando-se a posição de cada uma na arena e, dessa forma, a frequiência de contatos com ovos de ambos os hospedeiros e, no final de 5 dias, o número de ovos parasitados.

Os resultados referentes ao desenvolvimento de $T$. atopovirilia e $T$. pretiosum foram submetidos à análise de variância e as médias comparadas pelo teste de Tukey $(\mathrm{P} \leq 0,05)$. As médias relativas à capacidade de parasitismo e ao comprimento da tíbia posterior e do ovipositor foram comparadas pelo teste de Tukey ( $\mathrm{P} \leq 0,05)$, sendo os tratamentos dispostos em esquema fatorial com dois fatores, espécie de Trichogramma e hospedeiro de criação, adotando-se o delineamento experimental inteiramente casualizado. Foi feita uma análise de correlação para se verificar a relação entre o tamanho da tíbia posterior e o comprimento do ovipositor de T. atopovirilia e T. pretiosum. A preferência para oviposição entre ovos de S. frugiperda e A. kuehniella foi comparada pelo teste não paramétrico de $\chi^{2}$.

\section{RESULTADOSEDISCUSSÃO}

Não houve diferenças significativas para o período de desenvolvimento e a viabilidade de T. atopovirilia e $T$. pretiosum em ovos de $S$. frugiperda (Tabela I). A adequação dos ovos de $S$. frugiperda ao desenvolvimento de $T$. atopovirilia e T. pretiosum foi comprovada pelo período de desenvolvimento e alta viabilidade, que estão próximos às médias observadas para estas espécies criadas em outros hospedeiros, como mostram os resultados de HARRISON et al. (1985) e CôNSOlI \& PARRA (1996), que observaram para $T$. pretiosum criada em ovos de Heliothis virescens (Fabricius) um período de desenvolvimento de 10,7 e 10,3 dias e uma viabilidade de $99,3 \%$ e $94,2 \%$, respectivamente e de GREENBERG et al. (1988) que relataram um período de desenvolvimento e uma sobrevivência médios de 9,4 dias e 75,4\%, respectivamente, para T. pretiosum em ovos de Spodoptera 
exigua (Hübner).

A longevidade diferiu estatisticamente entre as espécies. Em média, fêmeas de T. pretiosum foram mais longevas do que as de T. atopovirilia (Tabela I); porém, para ambas as espécies os valores observados foram inferiores àqueles referidos por SÁ \& PARRa (1994) e Cônsoli \& PARRA (1996), para T. pretiosum criada em ovos de Helicoverpa zea (Boddie) e H. virescens, que foram de 9,9 e 16,0 dias, respectivamente e aos de RESENDE \& Ciociola (1996) para T. atopovirilia criada em ovos de $H$. zea (8 dias).

O número de adultos emergidos por ovo do hospedeiro foi significativamente maior para T. atopovirilia do que para $T$. pretiosum (Tabela I). Esses resultados concordam com os obtidos por NAVARRO \& MARCANO (1999) para T. atopovirilia e T. pretiosum mantidas em ovos de $H$. zea que foram de $1,4 \mathrm{e}$ 1,2 , respectivamente, sendo inferiores àqueles observados para T. pretiosum por HARRISON et al. (1985) em ovos de $H$. virescens e por SÁ \& PARRA (1994) em ovos de H. zea, a $25^{\circ} \mathrm{C}$ (3,2 e 2,2 adultos por ovos hospedeiro, respectivamente), devido ao maior tamanho dos ovos destas espécies. Assim, considerando-se que a capacidade de parasitismo (Tabela II) e o número de adultos emergidos por ovo foram maiores para T. atopovirilia do que para T. pretiosum, pode-se inferir que $T$. atopovirilia terá maior probabilidade de aumentar sua população em menor período de tempo do que T. pretiosum, sendo, aparentemente, mais adequada ao controle biológico de $S$. frugiperda.

SuZUKI et al. (1984) chamaram a atenção para a competição intraespecífica quando há uma maior quantidade de Trichogramma se desenvolvendo por ovo hospedeiro o que resultará em indivíduos de menor tamanho e de baixa qualidade. A fecundidade de Trichogramma está diretamente ligada ao seu tamanho, e este irá depender do número de parasitóides por ovo e do tamanho do hospedeiro (BAI et al. 1992; GreEnBerg et al. 1998). No entanto, os resultados desta pesquisa mostram que em ovos de S. frugiperda, há quantidade suficiente de nutrientes para suportar o desenvolvimento de mais do que um parasitóide por ovo hospedeiro, sem interferir na qualidade do inseto produzido.

A razão sexual não diferiu entre $T$. atopovirilia e $T$. pretiosum (Tabela I). Para ambas as espécies houve cerca de $70 \%$ de fêmeas, o que difere dos resultados de NAVARro \& Marcano (1999) para T. atopovirilia e T. pretiosum em ovos de $H$. zea, com 56 e $49 \%$ de fêmeas, respectivamente. A alta razão sexual observada para $T$. atopovirilia e $T$. pretiosum, embora possa ser uma característica inerente às duas espécies, devido à boa adequação dos ovos de S. frugiperda aos parasitóides, pode também estar relacionada ao tempo de parasitismo, 48 horas, que corresponde à fase inicial da vida adulta, época de maior atividade das fêmeas e na qual é produzido um maior número de fêmeas. Segundo estudos de Houseweart et al. (1983), Toonders \& Sánchez (1987) e HofFMAnN et al. (1995), fêmeas mais jovens tendem a colocar um maior número de ovos que darão origem a fêmeas, havendo uma inversão na razão sexual à medida que estas se tornam mais velhas. Assim, Hoffmann et al. (1995) constataram que
A

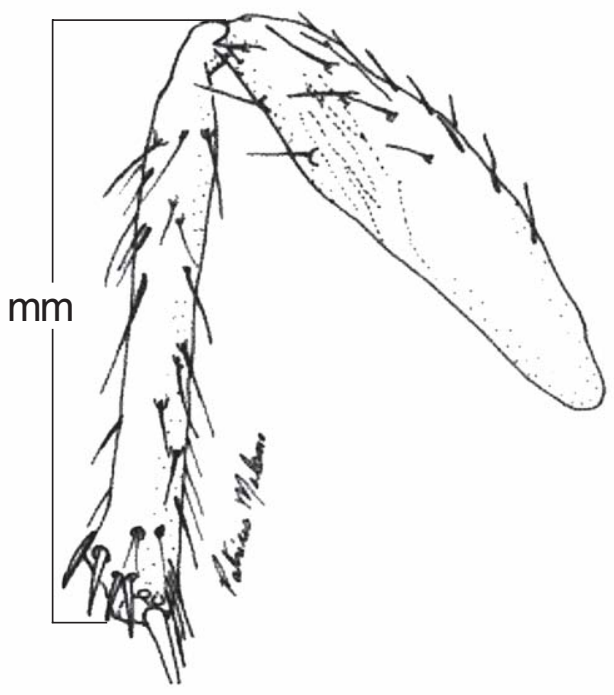

B

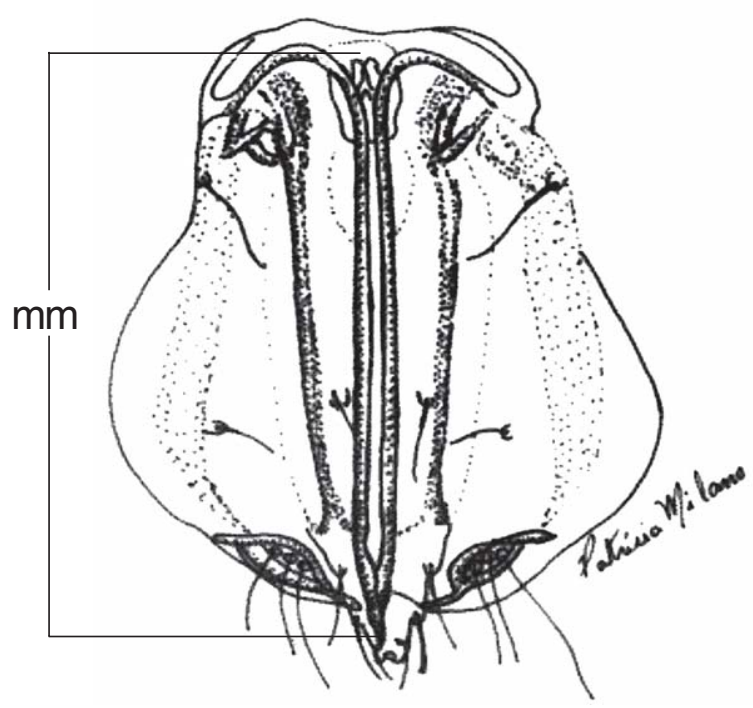

Fig. 1. Comprimento da tíbia posterior (A) e do ovipositor (B) de Trichogramma spp. (aumento de 500 vezes)

Trichogramma ostriniae Pang \& Chen colocou $80 \%$ de fêmeas durante os 6 primeiros dias de vida, diminuindo esta proporção com a idade da fêmea, e ToOnders \& SÁnchez (1987) observaram uma razão sexual com $50 \%$ de fêmeas para Trichogramma spp. em ovos de $S$. frugiperda, nos primeiros dias de parasitismo, caindo para $16 \%$ de fêmeas até o quinto dia de parasitismo. Uma outra hipótese para inversão na razão sexual com a idade da fêmea é, segundo HousEwEART et al. (1983), a diminuição ou a falta de esperma na espermateca, devido à fêmea copular uma única vez, o que diminuiria a fertilização dos ovos e, conseqüentemente, o número de fêmeas.

A capacidade de parasitismo de T. atopovirilia e $T$. pretiosum foi afetada pelo tipo de postura de $S$. frugiperda (Tabela II). Quanto ao número de ovos parasitados, houve interação significativa dos fatores espécie e tipo de postura, ou seja, a capacidade de parasitismo diferiu como resultado 

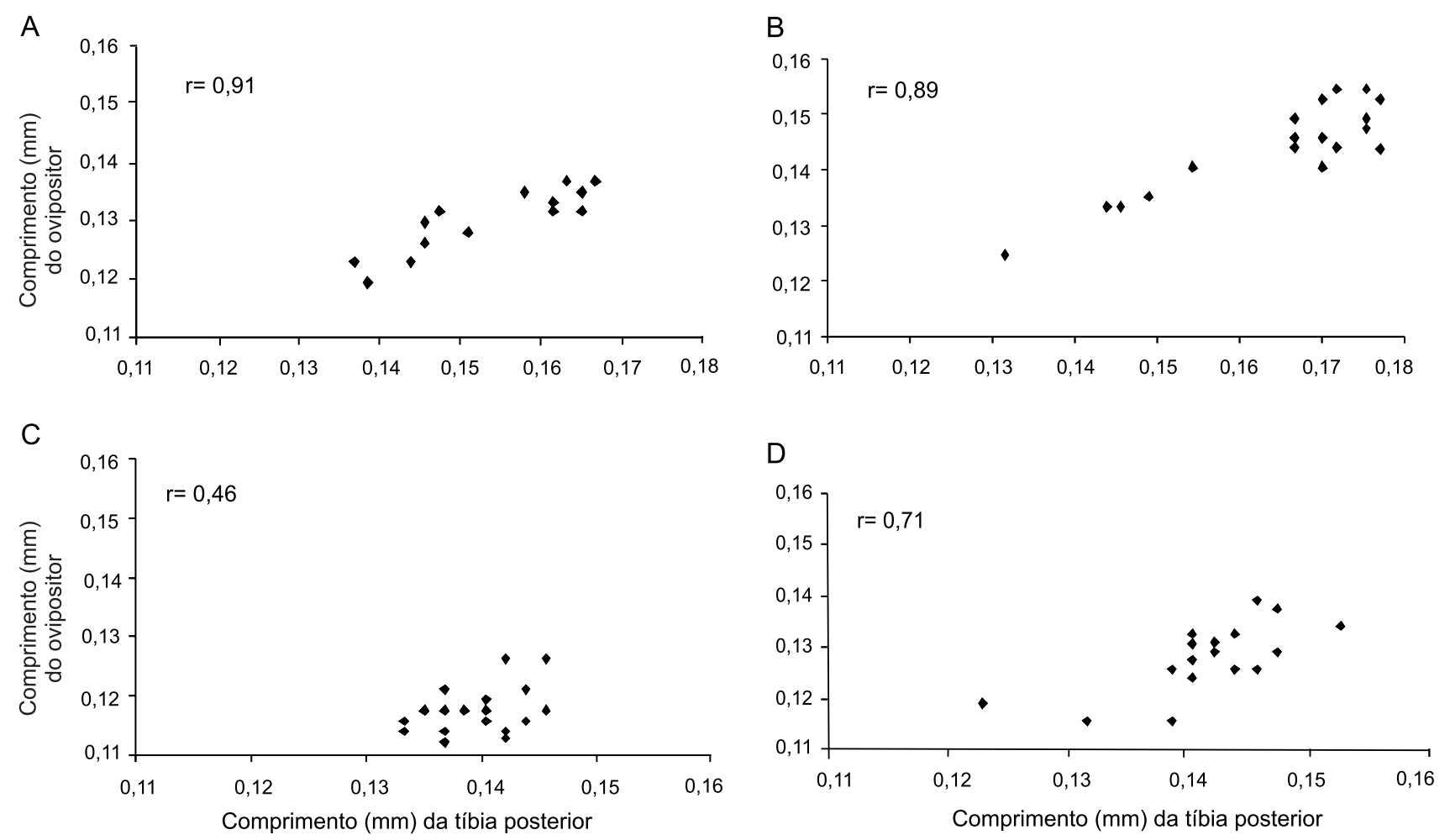

Fig. 2. Correlação entre o comprimento $(\mathrm{mm})$ da tíbia posterior e do ovipositor de duas espécies de Trichogramma. (A) T. atopovirilia e (B) T. pretiosum, criadas em ovos de S. frugiperda; (C) T. atopovirilia e (D) T. pretiosum, criadas em ovos de A. kuehniella.

da diferença de potencial reprodutivo entre as espécies e da dificuldade imposta ao parasitismo, pelas barreiras físicas das posturas de S. frugiperda. Quanto à porcentagem de parasitismo, houve diferença significativa entre as espécies e entre as posturas, mas não houve interação significativa destes fatores. Neste caso, a porcentagem de parasitismo independeu da interferência mútua dos fatores espécie e tipo de postura (Tabela II) e está relacionada à proporção entre o número de ovos por postura e a quantidade de ovos parasitados por $T$. atopovirilia e $T$. pretiosum.

O número de ovos parasitados por T. atopovirilia e $T$. pretiosum foi significativamente maior em posturas com uma e duas camadas sem escamas do que em posturas com escamas. Exceto em posturas com alta densidade de escamas, não houve diferença significativa quanto à porcentagem de parasitismo entre posturas com baixa densidade de escamas e sem escamas (uma e duas camadas) para as duas espécies (Tabela II). Esse resultado é consequiência da diferença entre o total de ovos por postura (que foi menor naquelas com baixa densidade de escamas do que nas posturas sem escamas) e o número de ovos parasitados, o que contribuiu para o aumento proporcional na porcentagem de ovos parasitados, não se detectando diferença significativa entre estas posturas (Tabela II). Contudo, a baixa capacidade de parasitismo observada em posturas com escamas mostra a dificuldade de Trichogramma em romper essa barreira e parasitar os ovos sobrepostos, diminuindo a eficiência dessas espécies no controle de $S$. frugiperda. Por outro lado, quando se observam os valores da capacidade de parasitismo em posturas com uma e duas camadas sem escamas, vê-se que estas não diferiram significativamente, indicando que a sobreposição não é um fator limitante nestes casos.

O número de ovos parasitados por T. atopovirilia foi significativamente maior do que por T. pretiosum, exceto em posturas com alta densidade de escamas (Tabela II). Este resultado mostra que, nessas posturas, a grande quantidade de escamas presentes impediu que houvesse um aumento no número de ovos parasitados, estando estes limitados aos ovos mais expostos na margem da postura, que provavelmente estavam em igual quantidade e abaixo da capacidade de parasitismo dessas duas espécies, o que contribuiu para a mesma proporção de ovos parasitados pelas duas espécies. Porém, a maior capacidade de parasitismo observada para $T$. atopovirilia difere dos resultados de NAVARRo \& MARCANO (1999) que constataram maior número de ovos de H. zea parasitados por T. pretiosum em relação a T. atopovirilia. Essa diferença na capacidade de parasitismo entre $T$. atopovirilia e T. pretiosum pode estar associada à preferência pelo hospedeiro e à maior fecundidade de T. atopovirilia em ovos de $S$. frugiperda, ou ainda, ao pequeno tamanho de T. pretiosum em relação à outra espécie em estudo.

Dessa forma, procurou-se verificar se a maior capacidade 
de parasitismo de T. atopovirilia em relação a T. pretiosum, estaria associada ao maior tamanho das fêmeas, o que facilitaria a quebra das barreiras físicas da postura por esta espécie e contribuiria para sua melhor performance. Observou-se interação significativa dos fatores espécie e hospedeiro de criação com relação ao comprimento da tíbia posterior, sendo o tamanho da fêmea dependente da espécie de Trichogramma e do hospedeiro no qual ela se desenvolveu. Quanto ao comprimento do ovipositor, só houve diferenças significativas quanto ao fator espécie e hospedeiro não havendo interação significativa destes fatores (Tabela III). O comprimento do ovipositor foi positivamente correlacionado com o comprimento da tíbia posterior de T. atoporivilia e T. pretiosum provenientes de S. frugiperda e A. kuehniella (Fig. 2), havendo uma relação direta entre tamanho da fêmea e o comprimento do ovipositor.

Fêmeas de $T$. atopivirilia e $T$. pretiosum tiveram maior comprimento da tíbia posterior e do ovipositor, quando desenvolvidas em ovos de $S$. frugiperda em relação àquelas criadas em ovos de A. kuehniella. A justificativa pode estar ligada ao fato dos ovos de S. frugiperda (volume de 0,036 $\mathrm{mm}^{3}$, segundo Cônsoli et al. 1999) serem maiores, com maior quantidade de nutrientes para permitir o desenvolvimento e produzir indivíduos de maior tamanho do que em ovos de $A$. kuehniella (volume de $0,023 \mathrm{~mm}^{3}$ segundo Cônsolı et al. 1999). Resultado semelhante foi observado por BAI et al. (1992) onde ovos de Manduca sexta (L.) de maior volume do que ovos de Trichoplusia ni (Hübner), produziram fêmeas de T. pretiosum de maior tamanho. Porém, Cônsoli \& PArra (1996) não encontraram diferenças significativas entre o comprimento da tíbia posterior de fêmeas de T. pretiosum criadas em ovos de H. zea, A. kuehniella, e in vitro, com valores de $0,156,0,157 \mathrm{e}$ $0,155 \mathrm{~mm}$, respectivamente.

Fêmeas de T. pretiosum provenientes de ovos de $S$. frugiperda tiveram maiores comprimentos da tíbia posterior do que fêmeas de T. atopovirilia, não havendo diferença, significativa, entre tais comprimentos, quando os insetos foram provenientes de ovos de A. kuehniella. O menor tamanho de T. atopovirilia pode ser explicado pelo maior número de indivíduos desenvolvendo-se por ovo de S. frugiperda (Tabela
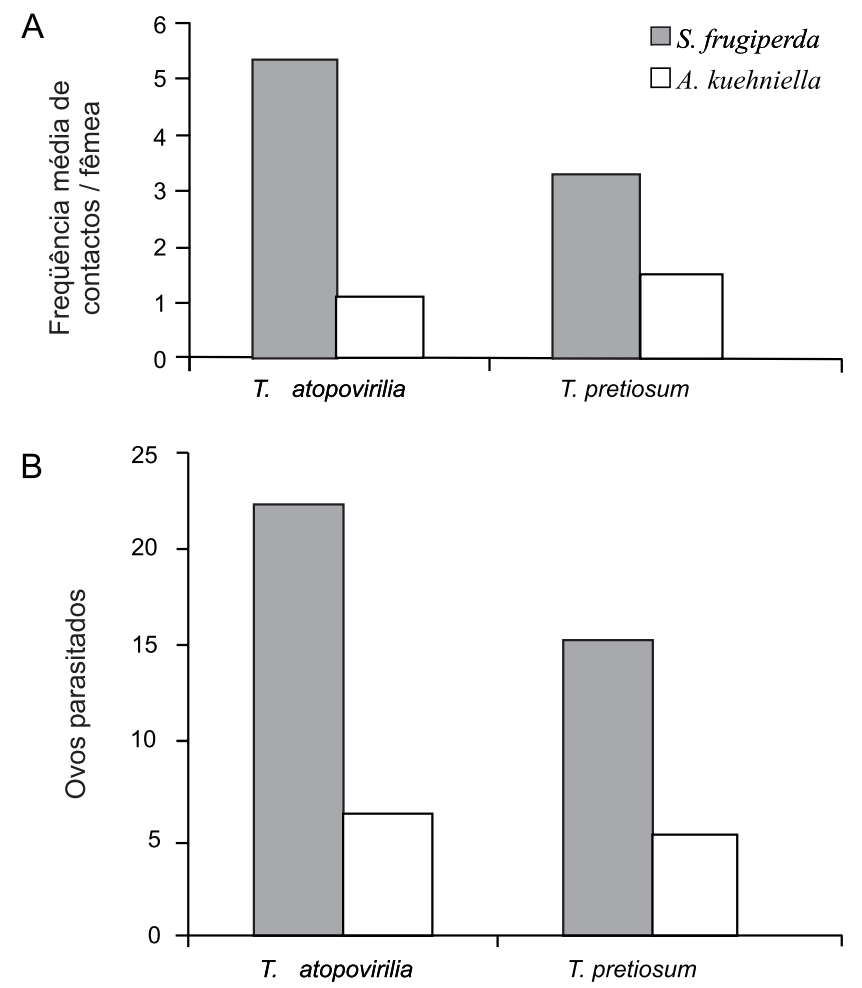

Fig. 3. Preferência de $T$. atopovirilia e $T$. pretiosum durante $45 \mathrm{~min}$, sobre ovos de $S$. frugiperda e A. kuehniella. Temperatura de $25 \pm 2^{\circ} \mathrm{C}$; U.R. de $70 \pm 10$ e fotofase de 14 h. (A) Frequiência média de contactos $\left(\chi^{2}=6,87, \mathrm{P}=0,09\right),(\mathrm{B})$ número médio de ovos parasitados $\left(\chi^{2}=2,15\right.$, $\mathrm{P}=0,14)$

I), pois, segundo WAAGE \& Ming (1984) existe uma relação inversa entre a densidade de Trichogramma por ovo hospedeiro e o tamanho do parasitóide.

Os valores encontrados para o tamanho da tíbia posterior de $T$. atopovirilia e $T$. pretiosum provenientes de ovos do hospedeiro natural, S. frugiperda, estão dentro do intervalo encontrado por Olson \& ANDOw (1998), para Trichogramma nubilale Pintureau \& Voegelé em ovos de Ostrinia nubilalis (Hübner), que variou de 0,14 a $0,18 \mathrm{~mm}$. Para T. pretiosum, o

Tabela I. Parâmetros biológicos de T. atopovirilia e T. pretiosum em ovos de S. frugiperda. Temperatura $25,0 \pm 2^{\circ} \mathrm{C}$, U.R. $70 \pm 10 \%$ e fotofase de 14 horas.

\begin{tabular}{ccccccc}
\hline \multirow{2}{*}{ Espécie } & \multicolumn{2}{c}{ Ovo-adulto } & & \multicolumn{3}{c}{ Fase adulta } \\
\cline { 2 - 3 } & $\begin{array}{c}\text { Duração } \\
(\text { dias })\end{array}$ & $\begin{array}{c}\text { Emergência } \\
(\%)\end{array}$ & & $\begin{array}{c}\text { Longevidade } \\
(\text { dias })\end{array}$ & $\begin{array}{c}\mathrm{N}^{\circ} \text { de } \\
\text { adultos/ovo }\end{array}$ & $\begin{array}{c}\text { Razão } \\
\text { sexual }\end{array}$ \\
\hline $\begin{array}{c}\text { T. atopovirilia } \\
(\mathrm{n}-38)\end{array}$ & $9,80 \pm 0,04 \mathrm{a}^{1}$ & $93,51 \pm 1,34 \mathrm{a}$ & & $5,47 \pm 0,50 \mathrm{~b}$ & $1,40 \pm 0,03 \mathrm{a}$ & $0,75 \pm 0,02 \mathrm{a}$ \\
$\begin{array}{c}\text { T. pretiosum } \\
(\mathrm{n}=40)\end{array}$ & $9,66 \pm 0,06 \mathrm{a}$ & $93,81 \pm 0,97 \mathrm{a}$ & & $7,37 \pm 0,61 \mathrm{a}$ & $1,27 \pm 0,03 \mathrm{~b}$ & $0,76 \pm 0,03 \mathrm{a}$ \\
\hline
\end{tabular}

${ }^{1}$ médias seguidas da mesma letra não diferem entre si pelo teste de "F" $(\mathrm{P}<0,05)$ 
Tabela II. Capacidade de parasitismo de T. atopovirilia e T. pretiosum em posturas de $S$. frugiperda com diferentes características físicas, em laboratório. Temperatura de $25 \pm 2,0^{\circ} \mathrm{C}$, U.R. $70 \pm 10 \%$ e fotofase de 14 horas.

\begin{tabular}{|c|c|c|c|c|c|}
\hline \multirow{3}{*}{ Tratamento } & \multirow{3}{*}{$\mathrm{n}$} & \multicolumn{4}{|c|}{ Capacidade de parasitismo } \\
\hline & & \multicolumn{2}{|c|}{$\mathrm{N}^{\mathrm{o}}$ de ovos parasitados } & \multicolumn{2}{|c|}{ Parasitismo (\%) } \\
\hline & & T. atopovirilia & T. pretiosum & T. atopovirilia & T. pretiosum \\
\hline Uma camada sem escamas & 15 & $\begin{array}{c}39,5 \pm 1,85 \mathrm{aA}^{1} \\
(\mathrm{n}=64,13)^{2}\end{array}$ & $\begin{array}{c}24,1 \pm 1,68 \mathrm{bA} \\
(\mathrm{n}=65,68)\end{array}$ & $63,2 \pm 3,73 \mathrm{~A}^{1}$ & $38,4 \pm 3,62 \mathrm{~A}$ \\
\hline Duas camadas sem escamas & 15 & $\begin{array}{c}38,4 \pm 2,02 \mathrm{aA} \\
(\mathrm{n}=70,13)\end{array}$ & $\begin{array}{c}23,2 \pm 1,78 \mathrm{bA} \\
(\mathrm{n}=75,13)\end{array}$ & $56,0 \pm 3,19 \mathrm{~A}$ & $32,1 \pm 2,76 \mathrm{~A}$ \\
\hline Uma camada e baixa densidade de escamas & 15 & $\begin{array}{c}26,9 \pm 1,76 \mathrm{aB} \\
(\mathrm{n}=55,93)\end{array}$ & $\begin{array}{c}15,9 \pm 1,31 \mathrm{bB} \\
(\mathrm{n}=53,40)\end{array}$ & $50,9 \pm 5,02 \mathrm{~A}$ & $33,6 \pm 4,37 \mathrm{~A}$ \\
\hline Uma camada e alta densidade de escamas & 15 & $\begin{array}{c}10,9 \pm 1,63 \mathrm{aC} \\
(\mathrm{n}=52,80)\end{array}$ & $\begin{array}{c}7,7 \pm 1,62 \mathrm{aC} \\
(\mathrm{n}=59,06)\end{array}$ & $23,1 \pm 3,56 \mathrm{~B}$ & $14,8 \pm 3,57 \mathrm{~B}$ \\
\hline Média Geral & 60 & $28,9 \pm 1,74 a$ & $17,7 \pm 1,16 b$ & $48,3 \pm 2,75 a$ & $29,7 \pm 3,57 b$ \\
\hline
\end{tabular}

comprimento da tíbia posterior observado neste estudo foi igual àqueles obtidos por BAI et al. (1992) e próximos aos resultados de Cônsoli \& PARRA (1996), para esta espécie proveniente de ovos de $H$. zea e A. kuehniella, que foram de 0,16 e $0,14 \mathrm{~mm}$ e 0,157 e $0,156 \mathrm{~mm}$, respectivamente.

O comprimento da tíbia posterior é uma medida que serve como parâmetro do tamanho da fêmea de Trichogramma, e se correlaciona positivamente com a capacidade dos machos localizarem as fêmeas para acasalar e com a capacidade de localização do hospedeiro, longevidade e fecundidade das fêmeas (WAAGe \& Ming 1984; BAi et al. 1992; Greenberg et al. 1998; Olson \& Andow 1998). Considerando-se que a capacidade de parasitismo de T. atopovirilia e T. pretiosum foi avaliada com espécies provenientes de ovos de $A$. kuehniella e que não houve diferença de tamanho entre fêmeas provenientes deste hospedeiro, os resultados mostram que a maior capacidade de parasitismo de T. atopovirilia não está associada ao seu tamanho como comentado anteriormente, mas à sua maior agressividade e especificidade a ovos de $S$. frugiperda, se comparado a T. pretiosum. Sendo assim, fica evidenciado que, em condições de laboratório, T. atopovirilia tem maior capacidade de parasitismo sobre posturas de $S$. frugiperda em relação a T. pretiosum. No entanto, ficou claro que é a densidade de escamas, e não o número de camadas, o fator mais importante neste parasitismo, já que esta funciona como uma barreira física que dificulta o parasitismo, mesmo para as espécies mais agressivas como T. atopovirilia.

A análise da freqüência de contactos entre ovos de $S$. frugiperda e A. kuehniella mostrou diferenças na preferência hospedeira entre $T$. atopovirilia e T. pretiosum, porém sem interação significativa dos fatores espécie e hospedeiro quanto ao número de ovos parasitados (Fig. 3). Em geral, a frequiência de contacto em ovos de $S$. frugiperda foi maior do que em ovos de A. kuehniella, contribuindo para o aumento no número de ovos de $S$. frugiperda parasitados por $T$. atopovirilia e T. pretiosum. Estudos feitos por HASSAN \& GuO

Tabela III. Comprimento $(\mathrm{mm})$ da tíbia posterior e do ovipositor de $T$. atopovirilia e $T$. pretiosum criados em ovos de $S$. frugiperda e A. kuehniella. Temperatura de $25,0 \pm 2,0^{\circ}$ C, U.R. $70 \pm 10 \%$ e fotofase de 14 horas.

\begin{tabular}{lcccccc}
\hline \multirow{2}{*}{ Hospedeiro } & $\mathrm{n}$ & \multicolumn{2}{c}{ Comprimento da tíbia $(\mathrm{mm})^{1}$} & & \multicolumn{2}{c}{ Comprimento do ovipositor $(\mathrm{mm})^{2}$} \\
\cline { 3 - 4 } \cline { 6 - 7 } S. frugiperda & 20 & $0,1521 \pm 0,0027 \mathrm{bA}^{3}$ & $0,1657 \pm 0,0030 \mathrm{aA}$ & & $0,1292 \pm 0,0016 \mathrm{~A}^{3}$ & $0,1446 \pm 0,0018 \mathrm{~A}$ \\
A. kuehniella & 20 & $0,1394 \pm 0,0008 \mathrm{aB}$ & $0,1418 \pm 0,0014 \mathrm{aB}$ & & $0,1178 \pm 0,0008 \mathrm{~B}$ & $0,1288 \pm 0,0015 \mathrm{~B}$ \\
\hline Média geral & & $0,1457 \pm 0,0017 \mathrm{~b}$ & $0,1537 \pm 0,0025^{\mathrm{a}}$ & & $0,1235 \pm 0,0012 \mathrm{~b}$ & $0,1367 \pm 0,0017 \mathrm{a}$ \\
\hline
\end{tabular}

${ }^{1,2}$ dados transformados em $\mathrm{x}^{2}$ e $\log \mathrm{x}$, respectivamente

${ }^{3}$ médias seguidas da mesma letra minúscula nas linhas e maiúscula nas colunas não diferem entre si pelo teste Tukey $(\mathrm{p}<0,05)$ 
(1991) e WÜHRER \& HASSAN (1993), mostraram a perda ou a diminuição na preferência pelo hospedeiro natural quando Trichogramma é criado por várias gerações sobre o hospedeiro alternativo o que, segundo HASSAN (1989), é um indicativo da perda de eficiência no campo. Assim, T. atopovirilia e $T$. pretiosum mantiveram a preferência pelo hospedeiro natural mesmo tendo sido criadas por várias gerações no hospedeiro alternativo, e mantiveram, portanto, a agressividade e eficiência de parasitismo em ovos de $S$. frugiperda.

A frequiência de contactos em ovos de $S$. frugiperda contribuiu para o aumento no número de ovos parasitados por T. atopovirilia e T. pretiosum, mostrando que existe uma relação direta entre freqüência de contacto e parasitismo. Porém, T. atopovirilia contactou mais e tendeu a parasitar mais ovos de $S$. frugiperda do que os de A. kuehniella se comparado a T. pretiosum. A explicação para esta diferença pode estar relacionada à maior especificidade de T. atopovirilia a ovos de $S$. frugiperda, ou a uma diminuição na preferência pelo hospedeiro natural, como conseqüência do condicionamento pré-imaginal, resultante da criação de $T$. pretiosum no hospedeiro alternativo, o que é corroborado por WÜHRER \& HASSAN (1993); estes autores não observaram correlação entre a freqüência de contactos e parasitismo e detectaram uma diminuição na preferência de $T$. pretiosum por ovos de Plutella xylostella L. após ser criada em ovos de Sitotroga cerealella (Olivier). Sendo assim, pode-se considerar que T. atopovirilia apresentou maior aceitação pelo ovos do hospedeiro natural, mostrando-se mais agressiva e com maior potencial para o controle de $S$. frugiperda do que T. pretiosum.

Agradecimentos. À CAPES/PICDT pela concessão de bolsa de estudos que permitiu a realização desta pesquisa; ao Prof. Dr. Carlos Tadeu dos S. Dias, do Departamento de Ciências Exatas da ESALQ/ USP, pela colaboração na análise estatística e à Bióloga Patrícia Milano pelos desenhos esquemáticos que ilustram este trabalho.

\section{REFERÊNCIAS}

Bai, B.; R. F. Luck; L. Foster; B. Stephens \& J. A. M. Janssen. 1992. The effect of host size on quality attributes of the egg parasitoid, Trichogramma pretiosum. Entomologia Experimentalis et Applicata 64: 37-48.

Bertels, A. 1970. Estudos da influência da umidade sobre a dinâmica de populações de lepidópteros, pragas do milho. Pesquisa Agropecuária Brasileira 5: 67-79.

Bleicher, E. \& J. R. P. Parra. 1989. Espécies de Trichogramma parasitóides de Alabama argillacea. Biologia de três populações. Pesquisa Agropecuária Brasileira 24: 929-940.

Botelho, P. S. M.; J. R. P. Parra; E. A. Magrini; M. L. Haddad \& L. C. L. Resende. 1995. Parasitismo de ovos de Diatraea saccharalis (Fabr., 1794) (Lep.: Pyralidae) por Trichogramma galloi Zucchi, 1988 (Hym.: Trichogrammatidae) em duas variedades de cana-de-açúcar conduzidas em dois espaçamentos de plantio. Revista Brasileira de Entomologia 39: 591-595.

Cônsoli, F. L. \& J. R. P. PARRA. 1996. Biology of Trichogramma galloi and T. pretiosum (Hymenoptera: Trichogrammatidae) reared in vitro and in vivo. Annals of the Entomological Society of America 89: 828-834.
Cônsoli, F. L.; E. W. Kitajima \& J. R. P. ParRa. 1999. Ultrastructure of the natural and factitious host eggs of Trichogramma galloi Zucchi and Trichogramma pretiosum Riley (Hymenoptera: Trichogrammatidae). International Journal of Insect Morphology and Embryology 28: 211-229.

Cortez, H. M. \& J. A. Trujillo. 1994. Incidencia del gusano cogollero y sus enemigos naturales en tres agrosistemas de maiz. Turrialba 44: $1-9$.

GreenberG, S. M.; D. A. NoRdLund \& Z. Wu. 1998. Influence of rearing host on adult size and oviposition behavior of mass produced female Trichogramma minutum Riley and Trichogramma pretiosum Riley (Hymenoptera: Trichogrammatidae). Biological Control 11: 4348.

Harrison, W. W.; E. G. King \& J. D. Ouzts. 1985. Development of Trichogramma exiguum and T. pretiosum at five temperature regimes. Environmental Entomology 14: 118-121.

Hassan, S. A. 1989. Selection of suitable Trichogramma strains to control the codling moth Cydia pomonella and the two summer fruit tortrix moths Adoxophyes orana, Pandemis heparana (Lep.: Tortricidae). Entomophaga 34: 19-27.

Hassan, S. A. 1997. Seleção de espécies de Trichogramma para o uso em programas de controle biológico, p. 183-206. In: J. R. P. PARRA \& R. A. Zucchi (ed.). Trichogramma e o Controle biológico aplicado. Piracicaba, FEALQ, 324 p.

Hassan, S. A. \& M. F. Guo. 1991. Selection of effective strains of egg parasites of the genus Trichogramma (Hym., Trichogrammatidae) to control the European corn borer Ostrinia nubilalis Hb. (Lep., Pyralidae). Journal of Applied Entomology 111: 335-341.

Hoffmann, M. P.; D. L. Walker \& A. M. Shelton. 1995. Biology of Trichogramma ostriniae (Hym.: Trichogrammatidae) reared on Ostrinia nubilalis (Lep.: Pyralidade) and survey for additional hosts. Entomophaga 40: 387-402.

Houseweart, M. W.; D. T. Jennings; C. Welty \& S. G. Southard. 1983. Progeny production by Trichogramma minutum (Hymenoptera: Trichogrammatidae) utilizing eggs for Choristoneura fumiferana (Lepidoptera: Tortricidae) and Sitotroga cerealella (Lepidoptera: Gelechiidae). Canadian Entomology 115: 1245-1252.

Kazmer, D. J. \& R. F. LuCK. 1995. Field tests of the size-fitness hypothesis in the egg parasitoid Trichogramma pretiosum. Ecology 76: 412425 .

Navarro, R. \& R. Marcano. 1999. Preferencia de Trichogramma pretiosum Riley y T. atopovirilia Oatman y Platner por huevos de Helicoverpa zea (Boddie) de diferentes edades. Boletin de Entomologia Venezolana 14: 87-93.

Olson, D. M. \& D. A. ANDow. 1998. Larval crowding and adult nutrition effects on longevity and fecundity of female Trichogramma nubilale Ertle \& Davis (Hymenoptera: Trichogrammatidae). Environmental Entomology 27: 508-514.

Resende, D. L. M. C. \& A. I. Ciociola. 1996. Capacidade de parasitismo de Trichogramma atopovirilia Oatman \& Platner, 1983 (Hymenoptera: Trichogrammatidae) em ovos de Helicoverpa zea (Boddie) (Lepidoptera: Noctuidae) em diferentes temperaturas. Ciência e Agrotecnologia 20: 421-424.

SÁ, L. A. N. \& J. R. P. Parra. 1994. Biology and parasitism of Trichogramma pretiosum Riley (Hym., Trichogrammatidae) on Ephestia kuehniella (Zeller) (Lep., Pyralidae) and Heliothis zea (Boddie) (Lep., Noctuidae) eggs. Journal of Applied Entomology 118: $38-43$.

SснміDт, J. M. \& J. J. Sмітн. 1985. The mechanism by which the parasitoid wasp Trichogramma minutum responds to host clusters. Entomologia Experimentalis et Applicata 39: 287-294.

Suzuki, Y.; H. Tsuji \& M. Sasakawa. 1984. Sex allocation and effects of superparasitism on secondary sex ratios in the gregarious parasitoid, Trichogramma chilonis (Hymenoptera: Trichogrammatidae). Animal Behavior 32: 478-484.

Toonders, T. J. \& J. L. C. SÁnchez. 1987. Evaluación de la efectividad de Trichogramma spp. (Hymenoptera: Trichogrammatidae) en el combate de Spodoptera frugiperda (J.E. Smith) (Lepidoptera: Noctuidae) recomendaciones para su uso. Centro Entomologia y Acarologia, 75-84. 
WaAge, J. K. \& N. G. S. Ming. 1984. The reproductive strategy of a parasitic wasp. I. Optimal progeny and sex allocation in Trichogramma evanescens. Journal of Animal Ecology 53: 401415 .
Wührer, B. G. \& S. A. Hassan. 1993. Selection of effective species/ strains of Trcihogramma (Hym., Trichogrammatidae) to control the diamondback moth Plutella xylostella L. (Lep., Plutellidae). Journal of Applied Entomology 116: 80-89.

Recebido em 06.VI.2003; aceito em 30.X.2003 